\title{
Caracterização epidemiológica e fatores de risco associados à infecção por Chlamydophila abortus em ovinos deslanados do semiárido brasileiro ${ }^{1}$
}

\author{
Areano E.M. Farias ${ }^{2}$, Severino S.S. Higino ${ }^{2}$, Sergio S. Azevedo ${ }^{2 *}$, Diego F. Costa ${ }^{2}$, \\ Fabrine A. Santos ${ }^{2}$, Carolina S.A.B. Santos ${ }^{2}$, Rosa Maria Piatti ${ }^{3}$ e Clebert J. Alves ${ }^{2}$
}

\begin{abstract}
Farias A.E.M., Higino S.S.S., Azevedo S.S., Costa D.F., Santos F.A., Santos C.S.A.B., Piatti R.M. \& Alves C.J. 2013. [Epidemiological characterization and risk factors associated with Chlamydophila abortus infection in sheep in Brazilian semiarid.] Caracterização epidemiológica e fatores de risco associados à Infecção por Chlamydophila abortus em ovinos deslanados do semiárido brasileiro. Pesquisa Veterinária Brasileira 33(3):286290. Departamento de Medicina Veterinária Preventiva e Saúde Animal, Faculdade de Medicina Veterinária e Zootecnia, Universidade de São Paulo, Av. Prof. Dr. Orlando Marques de Paiva 87, São Paulo, SP 05508-270, Brazil. E-mail: ssazevedo@cstr.ufcg.edu.br

The aim of this investigation was to determine the flock-level and animal-level prevalences of Chlamydophila abortus in sheep from the semiarid region of Northeastern Brazil, as well as to identify risk factors. Blood samples were collected from 476 sheep of 72 flocks in 14 counties in the Sertão mesoregion, state of Paraíba. For the serological diagnosis of Chlamydophila abortus infection the complement fixation test (FC) was carried out. A flock was positive when presented at least one seropositive animal. From the 72 flocks, 38 $(52.8 \%)$ presented at least one seropositive sheep, and 94 (19.7\%) of the 476 animals were seropositive. Participation in animal expositions (odds ratio $=4.31 ; 95 \% \mathrm{CI}=1.80-10.35$; $\mathrm{p}=0.011$ ) was identified as risk factor. It is suggested that $C$. abortus infection is spread in sheep of the region, and based on the risk factor analysis sanitary control in animal expositions is recommended.
\end{abstract}

INDEXING TERMS: Chlamydophila abortus, small ruminants, epidemiology, semiarid of Brazil.

RESUMO.- 0 objetivo do presente trabalho foi determinar as prevalências de propriedades positivas e animais soropositivos para Chlamydophila abortus em ovinos deslanados da região semiárida do Nordeste do Brasil, bem como identificar fatores de risco. Foram colhidas amostras de sangue de 476 ovinos procedentes de 72 propriedades em 14 municípios na mesorregião do Sertão, Estado da Paraíba. Para o diagnóstico sorológico da infecção por Chlamydophila abortus foi utilizada a reação de fixação de complemento (RFC). Uma propriedade foi considerada positiva quando apresentou pelo menos um animal soropositivo. Das 72

\footnotetext{
${ }^{1}$ Recebido em 26 de novembro de 2012.

Aceito para publicação em 15 de janeiro de 2013.

${ }^{2}$ Unidade Acadêmica de Medicina Veterinária, Centro de Saúde e Tecnologia Rural (CSTR), Universidade Federal de Campina Grande (UFCG), Av. Universitária s/n, Patos, PB 58700-970, Brasil. *Autor para correspondência: ssazevedo@cstr.ufcg.edu.br

${ }^{3}$ Centro de Pesquisa e Desenvolvimento em Sanidade Animal, Instituto Biológico de São Paulo, Av. Conselheiro Rodrigues Alves 1252, São Paulo, SP 04014-002, Brasil.
}

propriedades usadas $38(52,8 \%)$ apresentaram pelo menos um animal soropositivo, e dos 476 animais 94 (19,7\%) foram soropositivos. Participação em exposições (odds ratio $=4,31$; IC $95 \%=1,80-10,35 ; \mathrm{p}=0,011$ ) foi identificada como fator de risco. Sugere-se que a infecção por Chlamydophila abortus encontra-se disseminada em ovinos da região, e baseando-se na análise de fatores de risco, recomenda-se o controle sanitário nas exposições de animais.

TERMOS DE INDEXAÇÃO: Chlamydophila abortus, pequenos ruminantes, epidemiologia, semiárido do Brasil.

\section{INTRODUÇÃO}

A ovinocultura brasileira destaca-se no cenário nacional por apresentar um grande potencial de crescimento, tendo-se observado, nos últimos anos, uma evolução significativa no rebanho nacional, contando hoje com um efetivo de ovinos que chega a mais de 17 milhões de cabeças, representando aumento de aproximadamente $10 \%$ entre os anos de 2005 a 2010 (Brasil 2009). Esse crescimento pode ser 
explicado por algumas vantagens da ovinocultura, como a necessidade de menor área de criação, menor consumo de alimentos, facilidade de manejo e grande diversidade de produção de carne e couro de boa qualidade, servindo como alternativa de renda (Fernandes 2009). Contudo, ainda é precário o desfrute do potencial produtivo desta atividade para o agronegócio nacional devido a questões produtivas tais como gerenciamento e articulações do setor primário da cadeia produtiva (Aro et al. 2007). Nesse contexto, as deficiências sanitárias envolvidas no processo evolutivo da ovinocultura brasileira necessitam de resolução; essas deficiências são representadas pelos prejuízos provocados pelas perdas associadas à ocorrência de doenças infecciosas (Fernandes 2009).

Nos ruminantes, as infecções por Chlamydophila abortus são reconhecidas como causas de prejuízos econômicos, principalmente em decorrência de distúrbios reprodutivos, além de apresentarem potencial zoonótico (Piatti et al. 2006). As bactérias da família Chlamydaceae acometem diversas espécies de animais, incluindo aves, ruminantes e outros mamíferos. Em ovinos, a infecção por $C$. abortus é denominada de Aborto Enzoótico e assume especial destaque devido a ocorrência de infertilidade, abortamento, natimortalidade, nascimento de animais fracos e aumento da reposição de animais nos rebanhos devido à ocorrência de abortos (Papp \& Shewen 1997, Longbottom \& Coulter 2003).

Em pequenos ruminantes, são poucos os estudos sorológicos para $C$. abortus conduzidos no Brasil. Piatti et al. (2006) observaram prevalência de $12 \%$ em 100 caprinos procedentes dos Estados de São Paulo, Mato Grosso, Minas Gerais e Bahia, porém, todos os 80 ovinos foram soronegativos. Pereira et al. (2009) relataram presença de anticorpos anti-Chlamydophila sp. Em 8,1\% (10/123) dos ovinos e em 12\% (20/167) dos caprinos no Estado de Pernambuco. Já Pinheiro-Júnior et al. (2010) determinaram prevalência de 21,5\% (59/274) para ovinos provenientes do Estado de Alagoas. Batista (2012) utilizaram 975 caprinos de uma bacia leiteira do semiárido paraibano e encontraram $91(9,3 \%)$ animais soropositivos. Até o momento, não há relatos de estudos conduzidos em ovinos do semiárido do Estado da Paraíba.

No semiárido paraibano, a ovinocultura representa uma das principais atividades econômicas, onde a venda de animais vivos e/ou peles constitui fonte adicional de recursos para obtenção de gêneros não produzidos na propriedade. Dessa maneira, o objetivo do presente trabalho foi determinar a prevalência de propriedades positivas (focos) e de animais soropositivos para $C$. abortus, bem como identificar os possíveis fatores de risco em ovinos deslanados do semiárido da Paraíba, utilizando amostragem planejada.

\section{MATERIAL E MÉTODOS}

O Estado da Paraíba é dividido geograficamente em quatro mesorregiões (Sertão Paraibano, Borborema, Agreste Paraibano e Mata Paraibana) e 23 microrregiões. A mesorregião do Sertão Paraibano possui como principal atividade a pecuária extensiva, $\mathrm{o}$ clima semiárido é predominante, com temperaturas médias anuais ultrapassando $26^{\circ} \mathrm{C}$, índice pluviométrico entre 500 e $300 \mathrm{~mm}$ anuais e vegetação característica de caatinga. No presente trabalho foram utilizados ovinos deslanados adultos provenientes da mesorregião do Sertão.

A amostragem foi delineada para a determinação da prevalência de propriedades positivas e de animais soropositivos. A amostragem das propriedades foi simples aleatória a partir de quadro amostral fornecido pelo Serviço de Defesa Animal do Estado da Paraíba. 0 número de propriedades a serem amostradas foi calculado com o programa EpiInfo versão 6.04 (Dean 1994), com o emprego dos seguintes parâmetros: prevalência esperada de propriedades positivas de 91,6\% (Pereira et al. 2009), nível de confiança de $99 \%$ e erro absoluto de 10\% (Thrusfield 2007). Para os cálculos, foi utilizada a fórmula para amostras simples aleatórias:

$$
\mathrm{n}=\frac{Z^{2} \times P(1-P)}{d^{2}}
$$

Onde:

$n=$ número de propriedades amostradas;

$Z=$ valor da distribuição normal para o nível de confiança de 99\%;

$P=$ prevalência esperada;

$d=$ erro absoluto.

Em seguida, o número de ovinos a serem selecionados foi determinado individualmente por rebanho para a detecção da presença da infecção, utilizando a seguinte fórmula (Thrusfield 2007):

$$
\mathrm{n}=\left[1-(1-p)^{\frac{1}{\mathrm{~d}}}\right] \times\left(N-\frac{\mathrm{d}}{2}\right)+1
$$

Onde:

$\mathrm{n}=$ tamanho da amostra;

p = probabilidade de detecção de pelo menos um animal soropositivo;

$\mathrm{N}=$ tamanho do rebanho;

$\mathrm{d}=$ número de animais soropositivos no rebanho.

A probabilidade de detecção de pelo menos um animal soropositivo no rebanho foi determinada no nível de confiança de $95 \%$ $(\mathrm{p}=0,95)$, e o número de animais soropositivos por rebanho (d) foi calculado assumindo prevalência intra-rebanho de 8,1\% (Pereira et al. 2009).

No total, foram utilizados 476 animais procedentes de 72 propriedades de 14 municípios da mesorregião do Sertão paraibano (Fig,1).

O trabalho de campo foi desenvolvido no período de julho de 2010 a julho de 2011. As atividades de campo incluíram a colheita de sangue, aplicação de questionário epidemiológico e envio para o Laboratório de Doenças Transmissíveis (LDT) do Centro de Saúde e Tecnologia Rural (CSTR) da Universidade Federal de Campina Grande (UFCG), em Patos, PB.

Para a pesquisa de anticorpos anti-Chlamydophila sp., foi empregada a microtécnica de Fixação de Complemento (OIE 2010). A reação foi realizada em microplacas utilizando-se soro teste nas diluições de 1:16 a 1:512. Utilizou-se como antígeno a cepa S26/3 de C. abortus na diluição 1:50 e o complemento na diluição correspondente a duas unidades fixadoras de complemento. 0 título de anticorpos foi considerado como a recíproca da maior diluição de soro que apresentou $50 \%$ de fixação do complemento. Amostras com título igual ou superior a 32 foram consideradas positivas e com título igual a 16 foram consideradas suspeitas.

Para a análise de possíveis fatores de risco associados com a soropositividade para a infecção por $C$. abortus foram utilizados os dados (Quadro 2) coletados em questionários epidemiológi- 


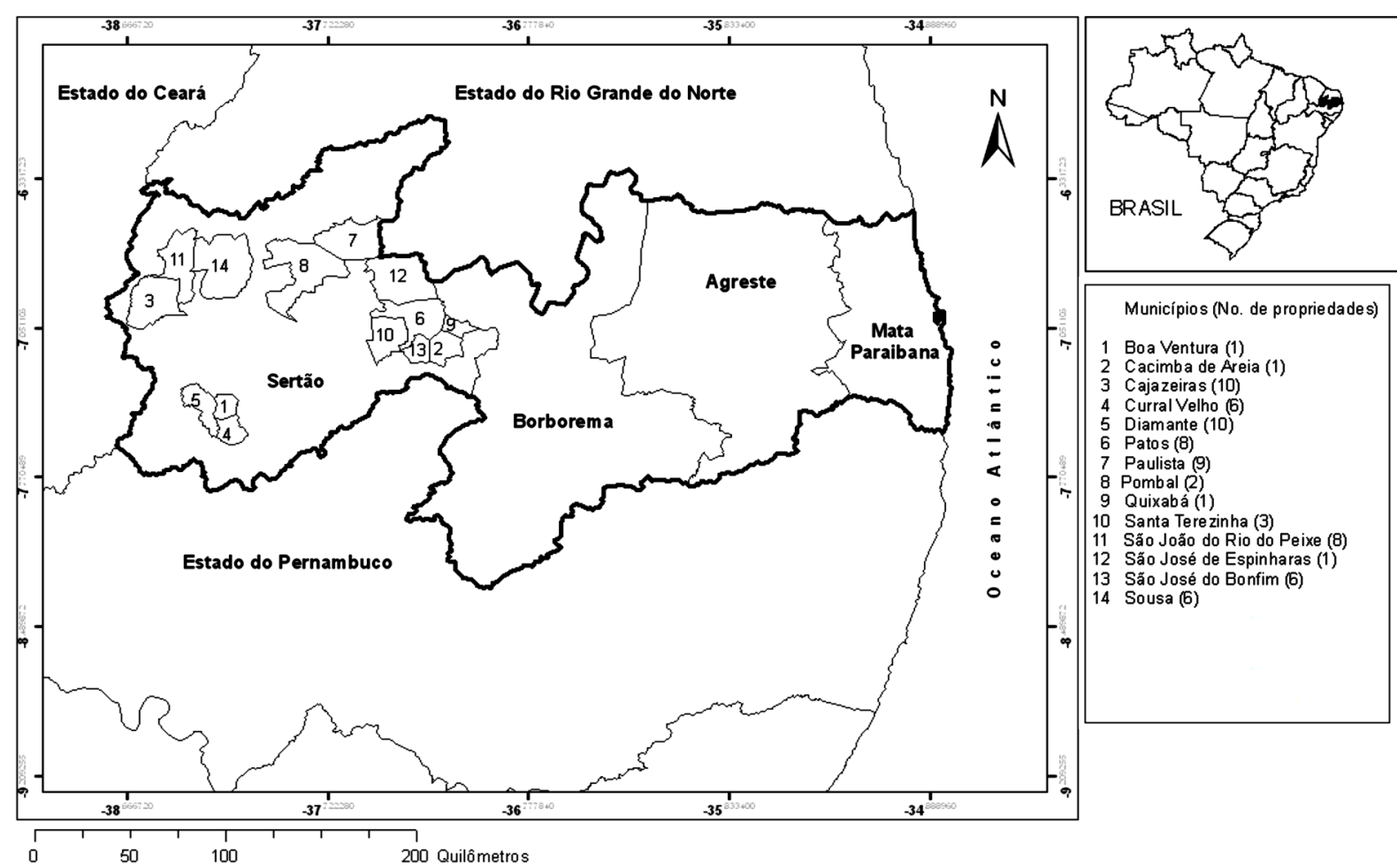

Fig.1. Estado da Paraíba como os municípios e respectivos números de propriedades rurais utilizadas, na mesorregião do Sertão paraibano.

Quadro 1. Prevalência de propriedades positivas (focos) e de animais soropositivos para a infecção por Chlamydophila abortus em ovinos deslanados do semiárido nordestino segundo o município, no período de julho de 2010 a julho de 2011

\begin{tabular}{|c|c|c|c|c|}
\hline \multirow[t]{2}{*}{ Município } & \multicolumn{2}{|c|}{$\begin{array}{l}\text { Prevalência por } \\
\text { propriedades }\end{array}$} & \multicolumn{2}{|c|}{$\begin{array}{c}\text { Prevalência por } \\
\text { animais }\end{array}$} \\
\hline & $\begin{array}{c}\text { № total de } \\
\text { proprie- } \\
\text { dades }\end{array}$ & $\begin{array}{c}\text { № de pro- } \\
\text { priedades po- } \\
\text { sitivas (\%) }\end{array}$ & $\begin{array}{l}\text { № total } \\
\text { de ani- } \\
\text { mais }\end{array}$ & $\begin{array}{c}\text { № de ani- } \\
\text { nais soropo- } \\
\text { sitivos }(\%)\end{array}$ \\
\hline Boa Ventura & 1 & $0(0,0)$ & 09 & $0(0,0)$ \\
\hline Cacimba de Areia & 1 & $0(0,0)$ & 05 & $0(0,0)$ \\
\hline Cajazeiras & 10 & $5(50,0)$ & 42 & $12(28,6)$ \\
\hline Curral Velho & 6 & $3(50,0)$ & 48 & $6(12,5)$ \\
\hline Diamante & 10 & $7(70,0)$ & 80 & $22(2,7)$ \\
\hline Patos & 8 & $4(50,0)$ & 43 & $9(20,9)$ \\
\hline Paulista & 9 & $7(77,8)$ & 79 & $18(22,8)$ \\
\hline Pombal & 2 & $1(50,0)$ & 18 & $1(5,5)$ \\
\hline Quixaba & 1 & $1(100,0)$ & 05 & $5(100,0)$ \\
\hline Santa Terezinha & 3 & $1(33,3)$ & 17 & $4(23,5)$ \\
\hline São João do Rio do Peixe & 8 & $2(25,0)$ & 48 & $3(6,25)$ \\
\hline São José de Espinharas & 1 & $1(100,0)$ & 03 & $2(66,6)$ \\
\hline São José do Bonfim & 6 & $3(50,0)$ & 39 & $5(12,8)$ \\
\hline Sousa & 6 & $3(50,0)$ & 40 & $7(17,5)$ \\
\hline TOTAL & 72 & $38(52,8)$ & 476 & $94(19,7 \%)$ \\
\hline
\end{tabular}

cos aplicados nas propriedades visitadas. A análise de fatores de risco foi conduzida em duas etapas: análise univariável e análise multivariável. Na análise univariável, cada variável independente foi cruzada com a variável dependente (condição sorológica dos animais), e aquelas que apresentaram valor de $\mathrm{p} \leq 0,20$ pelo teste de qui-quadrado (Zar 1999) foram selecionadas para a análise multivariável, utilizando-se regressão logística múltipla (Hosmer \& Lemeshow 2000). 0 nível de significância adotado na análise múltipla foi de $5 \%$. Todas as análises foram realizadas com o programa SPSS 20.0 for Windows.

\section{RESULTADOS E DISCUSSÃO}

De acordo com os dados obtidos no presente trabalho, $52,8 \%(38 / 72)$ das propriedades investigadas (Quadro 1) apresentaram pelo menos um ovino soropositivo para Chlamydophila abortus. Esta alta frequência sugere a possibilidade de disseminação do agente nos rebanhos de ovinos deslanados da região. Esses resultados são diferentes daqueles obtidos por Pereira et al. (2009), que encontraram $91,6 \%(11 / 12)$ de propriedades positivas, e dos obtidos por Pinheiro-Júnior et al. (2010), que encontraram frequência de $77,7 \%(21 / 27)$ de propriedades positivas. 0 principal ponto de divergência diz respeito às metodologias empregadas nesses trabalhos, nos quais foram utilizadas amostragens não-probabilísticas por conveniência, não permitindo, dessa forma, uma comparação consistente com os resultados do presente trabalho acerca da prevalência de propriedades-foco. Por outro lado, Batista (2012) utilizou amostragem planejada e investigou 110 propriedades de caprinos leiteiros, encontrando 55 propriedades com pelo menos um caprino soropositivo para C. abortus, revelando uma prevalência de $50 \%$, percentual este que se aproxima dos valores encontrados neste trabalho $(52,8 \%)$, reforçando o papel da infecção e a existência de fatores favorecedores para a disseminação do agente nos rebanhos ovinos da região, que de certa forma pode interferir na produtividade dos rebanhos, acarretando sérios prejuízos aos produtores. Os dados obtidos por Pinheiro-Júnior et al. (2010), no Estado de Alagoas, reforçam o papel da região do sertão, independente do clima, como um fator de risco para a doença, o que provavelmente está associado com a presença de maior quantidade dos efetivos ovinos e capri- 
nos na região quando comparado a outras regiões do pais, o que pode contribuir significativamente para a presença do agente e sua disseminação entre os rebanhos.

Dos 476 ovinos utilizados 94 foram soropositivos para C. abortus, resultando em prevalência de 19,7\%. Constata-se que poucos trabalhos no Brasil foram conduzidos com o objetivo de determinar a frequência de anticorpos contra C. abortus, fato este que preocupa, principalmente pela importância econômica que a atividade da caprino-ovinocultura representa para a região do semiárido brasileiro e os impactos provocados na esfera reprodutiva pelo agente. Os valores observados são superiores aos verificados por Pereira et al. (2009), 8,1\%; e por Piatti et al. (2006), que não detectaram ovinos soropositivos;porém, inferior aos de Pinheiro-Júnior et al. (2010), 21,5\%.

0 ponto de corte utilizado para caracterizar um animal como positivo foi título $\geq 32$. A distribuição dos títulos observados no presente estudo revela maior frequência de animais com título 32 (16,6\%; 79/476), seguido de animais com títulos 16 (15,5\%); 74/476), 64 (2,5\%; 12/476) e $128(0,6 \% ; 3 / 476)$. A frequência de animais suspeitos $(15,5 \%)$ encontrada foi inferior à observada por Pereira et al. (2009), 16,3\%; e superior a encontrada por Pinheiro-Júnior et al. (2010), 10,2\%. Conforme resoluções da Organização Mundial de Saúde Animal (OIE 2010), a Reação de Fixação de Complemento (RFC) é capaz de detectar anticorpos vacinais e infecções naturais. A similaridade antigênica entre C. abortus e C. pecorum pode explicar a ocorrência de possíveis resultados falso-positivos com títulos baixos. Dessa forma, títulos < 32 devem ser considerados inespecíficos para $C$. abortus. No entanto, na região objeto deste estudo, não se realiza a vacinação contra Chlamydophila sp., sendo somente praticada em algumas propriedades as vacinais anti-rábica e contra as clostridioses. No Brasil, inexistem relatos de infecção de pequenos ruminantes por C. pecorum (Pinheiro-Júnior et al. 2010), no entanto, esse agente é endêmico em ruminantes de vários países, sendo responsável por enfermidades inespecíficas: artrites, conjuntivite, enterite, encefalite, pneumonia e problemas reprodutivos (Iowa State University 2005, Longbottom \& Coulter 2003).

No tocante à análise de fatores de risco, as variáveis/ categorias mais associadas à ocorrência de animais soropositivos na análise univariável foram (Quadro 2): tipo de criação/extensiva/semi-intensiva $(p=0,056)$, finalidade da criação/reprodução $(\mathrm{p}=0,005)$, pastagem nativa/ausência $(\mathrm{p}=0,038)$, suplementação/fornece $(\mathrm{p}=0,026)$, aquisição frequente de animais/sim ( $\mathrm{p}=0,111)$, participação em exposições/sim $(p=0,001)$ e nascimento de crias mortas/sim $(\mathrm{p}=0,047)$. Na análise multivariável, o fator de risco identificado foi (Quadro 3) a participação em exposições (odds ratio $=4,31$; IC 95\% =1,80-10,35; $\mathrm{p}=0,011$ ).

A participação em exposições, apontada como fator de risco, reflete as condições de aglomerações de animais, que são sabidamente favoráveis à disseminação de agentes biológicos para os susceptíveis dentro das populações, sendo as chances cada vez maiores à medida que o número de animais e o tempo de exposição aumentam nestas populações (Thrusfield 2007). Destaca-se, ainda, a possibilidade do contato dos ovinos com restos placentários, fetos e descargas vaginais presentes no ambiente, o que reforça os argumentos de Silva et al. (2006), quando destacaram a importância do agente associado aos problemas reprodutivos, particularmente nas espécies de produção. Não se deve descartar a atividade de C. abortus nos rebanhos ovinos da região, fato este já mencionado por Pereira et

Quadro 2. Análise univariada dos possíveis fatores de risco associados á infecção por Chlamydophila abortus em ovinos deslanados do semiárido nordestino, no período de julho de 2010 a julho de 2011

\begin{tabular}{|c|c|c|c|c|c|}
\hline Variável & $\begin{array}{r}\text { № total d } \\
\text { animais }\end{array}$ & $\begin{array}{l}\text { Animais sor } \\
\text { positivos }(\%\end{array}$ & $\begin{array}{l}\text { Odds } \\
\text { ratio }\end{array}$ & IC 95\% & $\mathrm{P}$ \\
\hline \multicolumn{6}{|l|}{ Tipo de criação* } \\
\hline Intensiva & 4 & $0(0,0)$ & 1 & & \\
\hline Extensiva/semi-intensiva & 472 & $94(19,7)$ & $-\mathrm{a}$ & $-{ }^{a}$ & 0,056 \\
\hline \multicolumn{6}{|l|}{ Finalidade da criação* } \\
\hline Cria & 101 & $13(12,9)$ & 1 & & \\
\hline Recria/engorda & 247 & $47(19,0)$ & 1,59 & $0,79-3,27$ & 0,221 \\
\hline Reprodução & 65 & $21(32,3)$ & 3,23 & $1,39-7,60$ & 0,005 \\
\hline Subsistência & 63 & $13(20,6)$ & 1,76 & $0,70-4,43$ & 0,269 \\
\hline \multicolumn{6}{|l|}{ Tipo de exploração } \\
\hline Leite & 17 & $3(17,6)$ & 1 & & \\
\hline Corte & 371 & $70(18,9)$ & 1,09 & $0,28-4,89$ & 1,000 \\
\hline Outros & 88 & $21(23,9)$ & 1,46 & $0,34-7,12$ & 0,757 \\
\hline \multicolumn{6}{|l|}{ Criação tecnificada } \\
\hline Não & 387 & $73(18,9)$ & 1 & & \\
\hline Sim & 89 & $21(23,6)$ & 1,33 & $0,74-2,38$ & 0,388 \\
\hline \multicolumn{6}{|c|}{ Principal atividade da propriedade } \\
\hline Não & 383 & $72(18,8)$ & 1 & & \\
\hline Sim & 93 & $22(23,7)$ & 1,34 & $0,75-2,38$ & 30,363 \\
\hline \multicolumn{6}{|l|}{ Tamanho do rebanho } \\
\hline Até 18 & 124 & $24(19,4)$ & 1,10 & $0,61-1,99$ & 0,840 \\
\hline $19-31$ & 235 & $42(17,9)$ & 1 & & \\
\hline$>31$ & 117 & $28(23,9)$ & 1,45 & $0,81-2,57$ & 0,230 \\
\hline \multicolumn{6}{|l|}{ Contato com outros animais } \\
\hline Não & 197 & $37(18,8)$ & 1 & & \\
\hline Sim & 279 & 57( & 1,11 & 0,68 & 0,743 \\
\hline \multicolumn{6}{|l|}{ Pastagem nativa* } \\
\hline Ausência & 27 & $10(37,0)$ & 2,56 & $1,05-6,16$ & 0,038 \\
\hline Pres & 449 & $84(18,7)$ & 1 & & \\
\hline \multicolumn{6}{|l|}{ Suplementação* } \\
\hline Não fornece & 244 & $38(15,6)$ & 1 & & \\
\hline Fornece & 232 & $56(24,1)$ & 1,72 & $1,06-2,80$ & 0,026 \\
\hline \multicolumn{6}{|c|}{ Aquisição frequente de animais* } \\
\hline Não & 194 & $31(16,0)$ & 1 & & \\
\hline Sim & 282 & $63(22,3)$ & 1,51 & $0,92-2,50$ & 0,111 \\
\hline \multicolumn{6}{|c|}{ Participação em exposições* } \\
\hline Não & 454 & $83(18,3)$ & 1 & & \\
\hline Sim & 22 & $11(50,0)$ & 4,47 & 1,73-11,53 & 30,001 \\
\hline \multicolumn{6}{|l|}{ Vermifugações } \\
\hline Não & 49 & $9(1$ & 1 & & \\
\hline Sim & 427 & $85(19,9)$ & 1,10 & $0,49-2,55$ & 0,947 \\
\hline \multicolumn{6}{|l|}{ Presença de abortos } \\
\hline Não & 331 & $64(19,3)$ & 1 & & \\
\hline Sim & 145 & $30(20,7)$ & 1,09 & $0,65-1,82$ & 0,829 \\
\hline \multicolumn{6}{|c|}{ Nascimento de crias mortas* } \\
\hline Não & 350 & $61(17,4)$ & 1 & & \\
\hline Sim & 126 & $33(26,2)$ & 1,68 & $1,01-2,80$ & 0,047 \\
\hline \multicolumn{6}{|c|}{ Morte dos cordeiros nas primeira 24 horas } \\
\hline Não & 354 & $71(20,1)$ & 1,08 & $0,62-1,89$ & 0,876 \\
\hline Sim & 122 & $23(18,9)$ & 1 & & \\
\hline \multicolumn{6}{|l|}{ Morte após desmame } \\
\hline Não & 395 & $74(18,7)$ & 1 & & \\
\hline Sim & 81 & $20(24,7)$ & 1,42 & $0,78-2,59$ & 0,283 \\
\hline
\end{tabular}

* Variáveis selecionadas para a análise múltipla ( $\mathrm{p} \leq 0,20$ ou 0,15 ); ${ }^{a} \mathrm{Im}$ possível calcular a odds ratio e o IC $95 \%$ devido a um dos valores ter sido zero. 
Quadro 3. Fatores de risco associados á infecção por Chlamydophila abortus em ovinos deslanados do semiárido nordestino segundo o município, no período de julho de 2010 a julho de 2011, estimados por regressão logística múltipla

\begin{tabular}{cccc}
\hline Fator de risco & Odds ratio & IC 95\% & Valor de $\mathrm{p}$ \\
\hline Participação em exposições & 4,31 & $1,80-10,35$ & 0,011
\end{tabular}

al. (2009) e Pinheiro-Júnior et al. (2010). Outro relato que merece destaque diz respeito ao fato de que as infecções neonatais são a principal causa da perda de cordeiros no semiárido paraibano (Nóbrega Jr et al. 2005).

Neste particular, variáveis/categorias identificadas como possíveis fatores de risco na análise univariável sem, contudo, serem apontadas na análise multivariável, citadas neste trabalho, como finalidade de criação de reprodução $(\mathrm{p}=0,005)$, ausência de pastagem nativa $(\mathrm{p}=0,038)$ e fornecimento de suplementação $(\mathrm{p}=0,026)$, podem ajudar a entender esta situação e reforçar a hipótese da associação multifatorial. A finalidade de criação de reprodução reflete as condições mais rudimentares de tais propriedades, como a ausência de assistência veterinária frequente e a prática corriqueira de prender os animais durante a noite em instalações com alta concentração de animais. As vias de eliminação do agente mais comuns para ovinos são abortos, placenta e descargas vaginais (Silva et al. 2006), e isso, somado a grande aglomeração de animais, favorece a maior exposição entre os mesmos. Nóbrega Jr et al. (2005) já afirmaramque esse tipo de prática e a ausência de cuidados neonatais adequados favorecem a ocorrência de infecções neonatais entre os susceptíveis. Animais de propriedades com pastagem nativa ausente são alimentados com forragem e ração oriundas de outras propriedades ou cultivadas na mesma. Nas criações de ovinos estudadas não foram observadas segregação de animais por faixa etária, com os animais compartilhando no mesmo espaço bebedouros e cocho. Dessa forma, a água parada e alimentos contaminados por restos de aborto podem servir como vias de transmissão nos rebanhos (Pinheiro- Júnior et al. 2010). Com relação à associação entre fornecimento de suplementação e prevalência de animais soropositivos, sabe-se de fato que, na prática, as propriedades em estudo não suplementavam seus animais de modo adequado e, entre outros aspectos, a desnutrição, estresse e deficiências minerais também podem interferir na susceptibilidade dos animais aos processos infecciosos (Nóbrega Jr et al. 2005, Batista 2012).

\section{CONCLUSÃO}

Sugere-se que a infecção por Chlamydophila abortus encontra-se disseminada em ovinos do Sertão paraibano, e baseando-se na análise de fatores de risco, recomenda-se o controle sanitário nas exposições de animais.
Agradecimentos.- À Coordenação de Aperfeiçoamento de Pessoal de Nível Superior(CAPES) pela concessão de bolsa de Mestrado a Areano E.M. Farias.

\section{REFERÊNCIAS}

Aro D.T., Polizer K.A. \& Pena S.B. 2007. 0 agronegócio na ovinocultura de corte no Brasil. Rev. Cient. Eletr. Med. Vet. 3(7):1-6.

Batista C.S.A. 2012. Avaliação epidemiológica de agentes infecciosos e parasitários da esfera reprodutiva em caprinos leiteiros do semiárido da Paraíba. Tese de Doutorado em Ciências, Faculdade de Medicina Veterinária e Zootecnia, Universidade de São Paulo, São Paulo, SP. 86p.

Brasil 2009. Sistema Instituto Brasileiro de Geografia e Estatística (IBGE) de Recuperação Automática, SIDRA. Disponível em <http://www.sidra. ibge.gov.br/bda/>. Acessado em 26 jul. 2012.

Dean A.G. 1994. Epiinfo version 6: a word-processing, database, and statistic program for public health on IBM-compatible microcomputers. Center for Diseases Control and Prevention, Atlanta. 601p.

Fernandes C.E. 2009. Papel do ovino na cadeia epidemiológica da leptospirose pela Leptospira spp. sorovar Hardjo: fatores de risco que envolvem a infecção e transmissão entre ovinos e bovinos. Dissertação de Mestrado em Sanidade, Segurança Alimentar e Ambiental no Agronegócio, Instituto Biológico, São Paulo, SP. 101p.

Hosmer D.W. \& Lemeshow S. 2000. Applied Logistic Regression. $2^{\text {nd }} e d$. John Wiley and Sons, New York.

Iowa State University, Center for Food Security and Public Health 2005. Zoonotic Chlamydiae from Mammals. Available at <http://www.cfsph. iastate.edu/Factsheets/pdfs/!replaced/!chlamydiosis.pdf $>$ Accessed August 13, 2012.

Longbottom D. \& Coulter L.J. 2003. Animal chlamydioses and zoonotic implications. J. Comp. Pathol. 128(4):217-244.

Nóbrega Jr. J.E., Riet-Correa F., Nóbrega R.S., Medeiros J.M., Vasconcelos J.S., Simões S.V.D. \& Tabosa I.M. 2005. Mortalidade perinatal de cordeiros no semi-árido da Paraíba. Pesq. Vet. Bras. 25(3):171-178.

OIE 2010. Enzootic abortion in ewes (ovine chlamydiosis). Manual of Standards for Diagnostic Tests and Vaccines for Terrestrial Animals. World Organization for Animal Health. Available at <http://www.oie. int/eng/normes/mmanual> Accessed July 22, 2012.

Papp J.R. \& Shewen P.E. 1997. Chlamydia psittaci infection in sheep: a paradigm for human reproductive tract infection. J. Reprod. Immunol. 34(3):185-202.

Pereira M.F., Peixoto R.M., Piatti R.M., Medeiros E.S., Mota I.O., Azevedo S.S. \& Mota R.A. 2009. Ocorrência e fatores de risco para Chlamydophila abortus em ovinos e caprinos no estado de Pernambuco. Pesq. Vet. Bras. 29(1):33-40.

Piatti R.M., Scarcelli E.P. \& Genovez M.E. 2006. Pesquisa de anticorpos anti-Chlamydophila em caprinos e ovinos. Biológico, São Paulo, 68(2):138140.

Pinheiro Jr J.W., Mota R.A., Piatti R.M., Oliveira A.A.F., Silva A.M., Abreu S. R.O., Anderlini G.A. \& Valença R.M.B. 2010. Seroprevalence of antibodies to Chlamydophila abortus in ovine in the State of Alagoas, Brazil. Braz. J. Microbiol. 41(2):358-364.

Silva F.G., Freitas J.C. \& Muller E.E. 2006. Chlamydophila abortus em animais de produção. Ciência Rural 36(1):342-348.

Thrusfield M. 2007. Veterinary Epidemiology. $3^{\text {rd }}$ ed. Blackwell Science, Oxford.

Zar J.H. 1999. Biostatistical Analysis. $4^{\text {th }}$ ed. Prentice Hall, Upper Saddle River. 